Pacific Journal of Mathematic 


\title{
ON DETERMINING REGULAR BEHAVIOR FROM THE RECURRENCE FORMULA FOR ORTHOGONAL POLYNOMIALS
}

\author{
DANIEL P. MAKI
}

Ullman and Erdös and Freud have studied the distribution of the zeros of certain classes of orthogonal polynomials. Among other results they have shown that for a wide class of weight functions the associated orthogonal polynomials all have the same limiting zero distribution. We show, in a related result, that in certain cases one can deduce the limiting distribution of the zeros of the orthogonal polynomials without explicitly knowing the weight function (or the distribution function) of the orthogonal polynomials. In particular, for polynomials with certain types of triple recurrence formula we show that the limiting zero distribution is always the one studied by Ullman and Erdös and Freud. Polynomials with this limiting zero distribution are said to have "regular zero behavior".

In $\S 2$ we give the basic definitions and notation needed for our result. Our main theorem is in $\S 3$, and $\S 4$ contains some related comments and examples.

2. Definition and notation. We adopt the notation of Erdös and Freud in [3].

Let $d \alpha(x)$ be a nonnegative measure on $(-\infty, \infty)$ for which all moments

$$
\mu_{n}(d \alpha)=\int_{-\infty}^{+\infty} x^{m} d \alpha(x)
$$

exist and are finite and $\mu_{0}(d \alpha)=1$. We consider the orthonormal polynomials

$$
p_{n}(d \alpha ; x)=\gamma_{n}(d \alpha) \prod_{k=1}^{n}\left[x-x_{k n}(d \alpha)\right]
$$

which satisfy $\gamma_{n}(d \alpha)>0$ and $\int_{-\infty}^{+\infty} p_{n} p_{m} d \alpha=\delta_{n m}$, where $\delta_{n m}$ is the Kronecker delta. We are also interested in the monic polynomials $\left\{q_{n}(x)\right\}$ defined by $q_{n}(x)=p_{n}(d \alpha ; x) / \gamma_{n}(d \alpha), n \geqq 0$. The polynomials $\left\{q_{n}(x)\right\}$ are orthogonal with respect to $d \alpha(x)$, and thus they obey a triple recurrence formula of the following form (see [6]):

$$
\begin{aligned}
& q_{n}(x)=\left(x-c_{n}\right) q_{n-1}-\lambda_{n} q_{n-2} \\
& q_{-1}(x) \equiv 0, \quad q_{0}(x) \equiv 1 \\
& c_{n} \text { real, } \lambda_{n+1}>0, \quad n=1,2,3, \cdots .
\end{aligned}
$$


The zeros $x_{k n}(d \alpha)$ of $p_{n}(d \alpha ; x)$ (and hence also of $q_{n}(x)$ ) are real and simple, and we assume that they are ordered so that $x_{1 n}(d \alpha)>$ $x_{2 n}(d \alpha)>\cdots>x_{n m}(d \alpha)$. To simplify notation we write $x_{k n}$ for $x_{k n}(d \alpha)$. Let us denote by $N_{n}(d \alpha, t)$ the number of integers $k$ for which

$$
x_{k n}-x_{n n} \geqq t\left[x_{1 n}-x_{n n}\right] \quad(0 \leqq t \leqq 1)
$$

holds. The distribution function of the zeros, when it exists, is defined to be the limit.

$$
\beta(t)=\lim _{n \rightarrow \infty} \frac{N_{n}(d \alpha)}{n}, \quad 0 \leqq t \leqq 1 .
$$

In [8] and [3] it is shown that for a wide class of measures $d \alpha(x), \beta(t)$ does exist; and, moreover, $\beta(t)$ is given by

$$
\beta_{0}(t)=\frac{1}{2}-\frac{1}{\pi} \arcsin (2 t-1) .
$$

In this case the values $\theta_{k n}=\arcsin x_{k n}$ are equidistributed in the sense of Weyl, the measure $d \alpha(x)$ is said to be an arcsine measure, and the polynomials $p_{n}(d \alpha, x)$ are said to have regular zero behavior.

In [4] Erdös and Turan considered polynomials orthogonal on $[-1,+1]$. They showed that if $d \alpha(x)=p(x) d x$, where $p(x)$ is a nonnegative integrable function on $[-1,+1]$ such that $p(x)>0$ except for a set of Lebesgue measure zero, then $d \alpha(x)$ is an arcsine measure. In [8] Ullman gives a quite complete discussion of arcsine measures which are absolutely continuous, and in [3] Erdös and Freud establish a number of results in cases where $d \alpha(x)$ is not absolutely continuous. In particular, they obtain the following:

THEOREM 2.1 (Erdös and Freud). The condition

$$
\limsup _{n \rightarrow \infty}\left[\gamma_{n-1}\right]^{1 / n-1}\left[x_{1 n}-x_{n n}\right] \leqq 4
$$

implies that $d \alpha$ is an arcsine measure. Moreover, (2.5) implies that

$$
\lim _{n \rightarrow \infty}\left[\gamma_{n-1}\right]^{1 / n-1}\left[x_{1 n}-x_{n n}\right]=4 .
$$

We are interested in relating regular zero behavior to the recurrent formula (2.2). Thus, if a polynomial set is given by (2.2), is it possible to decide if the polynomials have regular behavior using only (2.2) (i.e., without knowledge of $d \alpha(x)$ or of any of the zeros of $\left.p_{n}(d \alpha, x)\right)$ ? We give a partial answer to this question, exhibiting a class of polynomials which have regular zero behavior, and also noting a class which does not. To obtain our results, we 
need the following results by Blumenthal and Chihara:

THEOREM 2.2 (Blumenthal, see [1]). Let the polynomials $\left\{q_{n}(x)\right\}$ be given by (2.2) and suppose $\lim _{n \rightarrow \infty} c_{n}=c, \lim _{n \rightarrow \infty} \lambda_{n}=\lambda>0$, where $c$ and $\lambda$ are finite. Also, let $X=\left\{x_{k n} ; 1 \leqq k \leqq n, n=1,2, \cdots\right\}$. Then $X$ is dense in the interval $[\sigma, \tau]$ where

$$
\sigma=c-2 \sqrt{\lambda}, \quad \tau=c+2 \sqrt{\lambda} .
$$

To state the Chihara result we need two definitions.

DEFINITION 1. The true interval of orthogonality of the orthogonal polynomials $\left\{p_{n}(d \alpha, x)\right\}$ is the smallest interval which contains in its interior all zeros of these polynomials.

Definition 2. A sequence $\left\{a_{n}\right\}_{n=1}^{\infty}, 0 \leqq a_{n} \leqq 1$, is called a chain sequence if there are numbers $g_{n}, 0 \leqq g_{n} \leqq 1$, such that $a_{n}=$ $\left(1-g_{n-1}\right) g_{n}(n=1,2, \cdots)$.

Theorem 2.3 (Chihara, [2]). Let $(a, b)$ denote the true interval of orthogonality of the polynomials $\left\{q_{n}(x)\right\}$ defined by (2.2). Then a is the maximum value of $c$ for which $c_{n}>c$ and $\left\{\lambda_{n+1} /\left(c_{n}-c\right)\left(c_{n+1}-c\right)\right\}$ is a chain sequence and $b$ is the minimum value of $d$ for which $d>c_{n}$ and $\left\{\lambda_{n+1} /\left(d-c_{n}\right)\left(d-c_{n+1}\right)\right\}$ is a chain sequence. If such $a$ (b) does not exist, then a (b) is $-\infty(+\infty)$.

3. Determining regular behavior. We are now ready to prove our result about determining regular behavior from the recurrence formula (2.2). We first point out that the condition $c_{n}=0, n \geqq 0$, is well known to be equivalent to having a measure $d \alpha(x)$ which is symmetric about $x=0$. In such a case the zeros of $p_{n}(d \alpha, x)$ are also symmetric about $x=0$.

THEOREM 3.1. Let the polynomial sequence $\left\{q_{n}(x)\right\}$ be defined by (2.2) and suppose $c_{n}=0, n \geqq 0$. Also, suppose that $\left\{\lambda_{n}\right\}$ satisfies $0<\lambda_{n} \leqq \lambda, n \geqq 1$ and $\lim _{n \rightarrow \infty} \lambda_{n}=\lambda$. Then the polynomials $\left\{q_{n}(x)\right\}$ have regular zero behavior.

Proof. By Theorem 2.2 we know that the zeros of the polynomials $\left\{q_{n}\right\}$ are dense in the closed interval $[-2 \sqrt{\lambda}, 2 \sqrt{\lambda}]$. Also, by Theorem 2.1 we know that if $\lim _{n \rightarrow \infty}\left(\gamma_{n-1}\right)^{1 / n-1}\left[x_{1 n}-x_{n n}\right]=4$, then the polynomials $\left\{q_{n}(x)\right\}$ have regular zero behavior. Let $d \alpha(x)$ be the probability measure for which the polynomials $\left\{q_{n}\right\}$ are orthogonal. That such a measure must exist was established by Favard. 
Also, from the general theory of moments and orthogonal polynomials, it is known that $d \alpha(x)$ is essentially unique, and it has bounded support which is symmetric about $x=0$ (see [7]). Let the true interval of orthogonality of the polynomials $\left\{q_{n}\right\}$ be $(-A, A)$. Then, by the definition of a true interval of orthogonality $x_{n n} \rightarrow-A$ and $x_{1 n} \rightarrow A$ as $n \rightarrow \infty$. Thus our proof is complete if we can show that

$$
\lim _{n \rightarrow \infty}\left[\gamma_{n-1}\right]^{1 / n-1}=\frac{4}{2 A}
$$

We establish (3.1) in two steps, first showing $A=2 \sqrt{\lambda}$ and then showing

$$
\lim _{n \rightarrow \infty}\left[\gamma_{n-1}\right]^{1 / n-1}=\frac{1}{\sqrt{\lambda}} .
$$

To show that $A=2 \sqrt{\lambda}$ we use Theorem 2.3. Thus, if $(-A,+A)$ is the true interval of orthogonality for $\left\{q_{n}(x)\right\}$, then $-A$ is the largest value of $c$ such that $c<0$ (recall $c_{n} \equiv 0$ ) and $\left\{\lambda_{n+1} / c^{2}\right\}$ is a chain sequence. Equivalently, $A$ is the smallest value of $d$ such that $d>0$ and $\left\{\lambda_{n+1} / d^{2}\right\}$ is a chain sequence. Now, since $0<\lambda_{n} \leqq \lambda$, for all $n \geqq 1$, and $\lim _{n \rightarrow \infty} \lambda_{n}=\lambda$, we know from the general theory of chain sequences (see [9]) that $\left\{\lambda_{n+1} / 4 \lambda\right\}$ is a chain sequence. This follows because $0<\lambda_{n+1} / 4 \lambda \leqq 1 / 4$ and $\lambda_{n+1} / 4 \lambda \rightarrow 1 / 4$, as $n \rightarrow \infty$. Moreover, if $A$ is any number less than $2 \sqrt{\lambda}$, then $\lambda_{n+1} / A^{2} \rightarrow \lambda / A^{2}>1 / 4$, and hence $\lambda_{n+1} / A$ would not be a chain sequence. Thus $A=2 \sqrt{\lambda}$ is the smallest positive number such that $\lambda_{n+1} / A^{2}$ is a chain sequence, and therefore the true interval of orthogonality is $(-2 \sqrt{\lambda}, 2 \sqrt{\lambda})$.

Next, we note that from (2.1), (2.2) and the fact that the polynomials $p_{n}(d \alpha, x)$ are orthonormal, we have

$$
\gamma_{n}(d \alpha)=\left[\frac{1}{\lambda_{2} \lambda_{3} \cdots \lambda_{n+1}}\right]^{1 / 2} .
$$

Therefore,

$$
\left[\lambda_{n-1}(d \alpha)\right]^{1 /(n-1)}=\left[\frac{1}{\left(\lambda_{2} \lambda_{3} \cdots \lambda_{n+1}\right)^{1 / 2}}\right]^{1 /(n-1)} .
$$

The proof will be complete if we can show that $\left[\gamma_{n-1}\right]^{1 /(n-1)} \rightarrow 4 / 2 A=$ $4 / 4 \sqrt{\lambda}=1 / \sqrt{\lambda}$ as $n \rightarrow \infty$. Equivalently, we must show that

$$
\lim _{n \rightarrow \infty}\left[\prod_{i=1}^{n-1} \lambda_{i+1}\right]^{1 /(n-1)}=\lambda
$$

But $\lambda_{i}>0$ for all $i$ and $\lambda_{i} \rightarrow \lambda>0$ as $i \rightarrow \infty$ so (3.2) follows directly from basic results about limits of products. Thus our proof is 
complete and we see that the zeros of the polynomials $\left\{q_{n}\right\}$ (and hence $\left.p_{n}(d \alpha, x)\right)$ have regular behavior.

4. Remarks and examples.

1. In Theorem 3.1 the hypothesis $\lambda>0$ is necessary for regular zero behavior. This follows because if in (2.2) $c_{n}=0$ for $n \geqq 0$ and $\lambda_{n} \rightarrow 0 n \rightarrow \infty$, then, by Theorem 2.2, $\sigma=\tau=0$ and the interval $[\sigma, t]$ where the zeros are dense reduces to a single point, the origin. Since regular zero behavior requires that the zeros be dense over the entire true interval of orthogonality, we see that regular zero behavior cannot occur when $c_{n} \equiv 0$ and $\lambda_{n} \rightarrow 0$.

2. In Theorem 3.1 if the hypothesis $c_{n} \equiv 0$ is replaced by $c_{n} \equiv c$, the result still holds. The only change which occurs is that the true interval of orthogonality is translated from $[-2 \sqrt{\lambda}, 2 \sqrt{\lambda}]$ to $[c-2 \sqrt{\lambda}, c+2 \sqrt{\lambda}]$.

3. From Ullman's results on weight function [8] it follows that many classical polynomial sets such as the Jacobi polynomials have regular zero behavior. Theorem 3.1 also shows that the Jacobi polynomials (and many other known sets) have regular zero behavior because they satisfy (2.2) with $c_{n} \equiv 0,0<\lambda_{n} \leqq 1 / 4$ and $\lambda_{n} \rightarrow 1 / 4$ as $n \rightarrow \infty$. Moreover, from Theorem 3.1 we also know that rather "simple" (but not well known) sets have regular zero behavior, but we do not know if they correspond to a measure which is absolutely continuous and hence has a weight function. It would be of interest to know (in terms of 2.2) when regular zero behavior is associated with a weight function and when it is not.

4. In [5], using different methods, Nevai obtains a great deal of information relating the support of the measure $d \alpha(x)$ to properties of the coefficients in the three term recurrence formula (2.2). In particular (using the notation of $\S 1$ ), he shows the following:

THEOREM (page 23, Theorem 7 [5]). Let the support of $d \alpha(x)$ be compact and suppose $\lim _{n \rightarrow \infty} c_{n}=c$ exists. Then support $d \alpha(x)=$ $A \cup B, A \cap B=\varnothing$, where $A$ is a closed subset of the interval

(*) $\quad\left[c-2 \limsup _{n \rightarrow \infty} \sqrt{\lambda_{n+1}}, \quad c+2 \limsup _{n \rightarrow \infty} \sqrt{\lambda_{n+1}}\right]$,

$B$ is finite or denumerable, isolated, and the only two possible limit points of $B$ are the endpoints of the interval (*). Moreover, if $x \in B$, then $\alpha_{a c}+\alpha_{s}$ is constant near $x$ and $\alpha$ has an isolated jump at $x$; in addition, 


$$
B \subset\left[\inf _{n} c_{n}-2 \sup _{n} \sqrt{\lambda_{n}}, \quad \sup _{n} c_{n}+2 \sup _{n} \sqrt{\lambda_{n}}\right] .
$$

In the special case of Theorem $3.1 B=\varnothing$, and $A=[-2 \sqrt{\lambda}, 2 \sqrt{\lambda}]$, as noted above.

\section{REFERENCES}

1. T. S. Chihara, Orthogonal polynomials whose zeros are dense in intervals, J. Math. Anal. and App., 24, No. 2 (1968), 362-371.

2. - Chain sequences and orthogonal polynominals, Trans. Amer. Math. Soc., 104, No. 1 (1962), 1-16.

3. P. Erdös and G. Freud, On orthogonal polynomials with regularly distributed zeros, Proc. London Math. Soc. (3), 29 (1974), 521-537.

4. P. Erdös and P. Turan, On interpolation III, Ann. of Math., 41 (1940), 510-555.

5. P. Nevai, Memoir of A.M.S. \#213, American Mathematical Society, 1979.

6. G. Szëgo, Orthogonal polynomials, Coll. Pub. 23 (Amer. Math. Soc.), New York, 1939.

7. J. Schohat and J. Tamarkin, The problem of moments, Math. Sur. No. 1, Amer. Math. Soc., New York, 1943.

8. J. L. Ullman, On the regular behavior of orthogonal polynomials, Proc. London Math. Soc. (3) 24 (1972), 119-148.

9. H. S. Wall, Analytic Theory of Continued Fractions, Van Nostrand, New York, 1948.

Received July 13, 1978.

INDIAN UNIVERSITY

BLOOMINGTON, IN 47401 


\section{PACIFIC JOURNAL OF MATHEMATICS}

\section{EDITORS}

DoNALD BABBITT (Managing Editor)

University of Galifornia

Los Angeles, California 90024

HUgo RossI

University of Utah

Salt Lake City, UT 84112

C. C. MOORE AND ANDREW OGG

University of California

Berkeley, CA 94720
J. DugunduI

Department of Mathematics

University of Southern California

Los Angeles, California 90007

R. Finn and J. Milgram

Stanford University

Stanford, California 94305

\section{ASSOCIATE EDITORS}
E. F. BeCKenbaCh
B. H. NeumanN
F. WOLF
K. YoSHIDA

\section{SUPPORTING INSTITUTIONS}

UNIVERSITY OF BRITISH COLUMBIA

CALIFORNIA INSTITUTE OF TECHNOLOGY

UNIVERSITY OF CALIFORNIA

MONTANA STATE UNIVERSITY

UNIVERSITY OF NEVADA, RENO

NEW MEXICO STATE UNIVERSITY

OREGON STATE UNIVERSITY

UNIVERSITY OF OREGON
UNIVERSITY OF SOUTHERN CALIFONIA STANFORD UNIVERSITY UNIVERSITY OF HAWAII UNIVERSITY OF TOKYO UNIVERSITY OF UTAH WASHINGTON STATE UNIVERSITY UNIVERSITY OF WASHINGTON 


\section{Pacific Journal of Mathematics}

\section{Vol. 91, No. $1 \quad$ November, 1980}

Harvey Leslie Abbott, Extremal problems on nonaveraging and nondividing

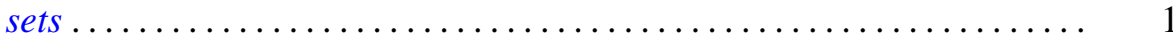

Marine Bruce Abrahamse and Stephen D. Fisher, Mapping intervals to



William Wells Adams, The best two-dimensional Diophantine



Marilyn Breen, A quantitative version of Krasnosel'skiu 's theorem in



Stephen LaVern Campbell, Linear operators for which $T^{*} T$ and $T T^{*}$

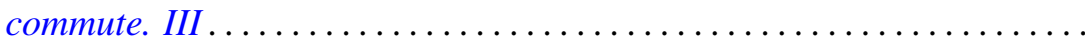

Zvonko Cerin, On cellular decompositions of Hilbert cube manifolds ......

J. R. Choike, Ignacy I. Kotlarski and V. M. Smith, On a characterization

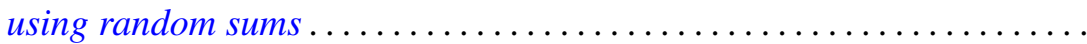

Karl-Theodor Eisele, Direct factorizations of measures .............. 79

Douglas Harris, Every space is a path component space ............. 95

John P. Holmes and Arthur Argyle Sagle, Analytic H-spaces, Campbell-Hausdorff formula, and alternative algebras.............

Richard Howard Hudson and Kenneth S. Williams, Some new residuacity criteria ..........................................

V. Karunakaran and Michael Robert Ziegler, The radius of starlikeness for a class of regular functions defined by an integral ....

Ka-Sing Lau, On the Banach spaces of functions with bounded upper

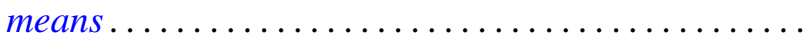

Daniel Paul Maki, On determining regular behavior from the recurrence formula for orthogonal polynomials................

Stephen Joseph McAdam, Asymptotic prime divisors and going down...

Douglas Edward Miller, Borel selectors for separated quotients ..

Kent Morrison, The scheme of finite-dimensional representations of an algebra

Donald P. Story, A characterization of the local Radon-Nikodým property by tensor products

Arne Stray, Two applications of the Schur-Nevanlinna algorithm ...

N. B. Tinberg, The Levi decomposition of a split $(B, N)$-pair ...

Charles Irvin Vinsonhaler and William Jennings Wickless, A theorem on quasi-pure-projective torsion free abelian groups of finite rank... 